\title{
Rapid Cardiac Adaptation to Exercise Demand Signal and Execution of Maximal Leg Muscle Contraction
}

\author{
Kiyomi TAKAYANAGI, Ishio NINOMIYA*, Osamu YOSHIMURA ${ }^{\dagger}$, and Issei SEYAMA ${ }^{\ddagger}$ \\ Department of Physical Therapy, School of Health Sciences, \\ Sapporo Medical University, Sapporo, 060-0063 Japan; \\ * Department of Clinical Engineering, Faculty of Health Sciences, \\ Hiroshima International University, Hiroshima, 724-0695 Japan; and \\ ${ }^{\dagger}$ Institute of Health Sciences and ${ }^{\ddagger}$ Department of Physiology, Faculty of Medicine, \\ Hiroshima University, Hiroshima, 734-8551 Japan
}

\begin{abstract}
We investigated the neural regulation of the cardiac interval to an exercise demand signal and to a repeated exercise in 20 healthy human subjects. Electrocardiogram (ECG), muscle torque, and electromyogram (EMG) were simultaneously measured and their time relationships compared before and during the exercise. The R-R interval of ECG was directly increased by the exercise demand signal itself before the onset of EMG but not reflexly by muscle contraction. The cardiac interval decreased at the onset of exercise. Under the condition of repeated maximum eccentric training, the resting cardiac interval decreased prior to the exercise, whereas
\end{abstract}

the brief increase in cardiac interval to the exercise demand signal remained unchanged. These results suggested that when autonomic nerve activity to the pacemaker is activated by the exercise demand signal, an initial effect of vagal nerve activity appears, and an effect of vagal nerve withdrawal and/or sympathetic nerve activity then appears. The responses of the heart and leg skeletal muscle at the onset of exercise are not synchronized, and the cardiac interval is controlled by vagal and sympathetic nerve activities to effect a transition to a high heart rate as quickly and smoothly as possible. [Japanese Journal of Physiology, 51, 709-716, 2001]

Key words: neural regulation system, high intensity exercise, cardiac adaptation, repeated training.

\begin{abstract}
According to experiments involving autonomic nerve blockades and spectral analyses of heart rates, the increase in the heart rate under light-intensity exercise is adjusted chiefly by the withdrawal of the vagal nerve activity [1-3]. The increase in the heart rate during submaximum intensity exercise is adjusted by a combination of increased sympathetic nerve activity and the withdrawal of vagal nerve activity [4-6]. However, the neural effect on rapid changes in cardiac interval at the onset of exercise cannot be observed by methods of autonomic nerve blockade or spectral analysis because a resting heart rate is increased or decreased by autonomic nerve blockade, and the transformation of some cardiac intervals from the time course data to the frequency data is necessary to
\end{abstract}

identify the autonomic nerve. Moreover, there has been very little discussion about the participation of the neural regulation system with regard to the instantaneous heart rate change at the onset of exercise in human [7].

In many experiments the cardiac interval at the onset of exercise was used as the origin of changes in cardiac interval during exercise, whereas the timing of onset of exercise was usually determined by the appearance of electromyogram (EMG). We observed decreases in the cardiac interval in many cases at the onset of exercise; in other cases, however, no change or even increases were found [8]. Such a wide variation of cardiac interval response at the onset of exercise indicates clearly that the change in cardiac inter-

Received on May 16, 2001; accepted on September 5, 2001

Correspondence should be addressed to: Kiyomi Takayanagi, Department of Physical Therapy, School of Health Sciences, Sapporo Medical University, South-3, West-17, Chuo-ku, Sapporo, 060-0063 Japan. Tel: +81-11-611-2111, Fax: +81-11-611-2150, E-mail: ktaka@sapmed.ac.jp 
val and the onset of exercise are independent of each other and that the change in cardiac interval was not induced by the exercise itself. We assumed that the change in cardiac interval was produced by the exercise demand signal.

In our previous experiment [8], the variation at the beginning of the cardiac interval response to the exercise seemed to depend on the timing of the exercise demand signal. Moreover, we perceived a paradoxical phenomenon in which in some subjects the cardiac interval just before or after the onset of maximal dynamic leg exercise was increased by the exercise demand signal itself, but not by muscle contraction. Therefore the goal of the present study was to investigate the phase effect of the exercise demand signal on the cardiac interval in detail and to discuss the participation of the neural regulation system with regard to the instantaneous change in heart rate to elucidate the relationship between autonomic nervous activity and somatic nervous activity at the onset of exercise. Another objective was to determine whether repeated unfamiliar eccentric leg exercise changed the paradoxical phenomenon of the cardiac interval and affected the time course and response magnitude of the cardiac interval.

\section{METHODS}

Subjects. Twelve female and 8 male college students participated in this study. Their mean age, height, and weight were $21.3 \pm 0.6$ (SE; standard error) years (range 19-24 years), $165 \pm 8 \mathrm{~cm}(150-178 \mathrm{~cm})$, and $61 \pm 6 \mathrm{~kg}(48-73 \mathrm{~kg})$, respectively. All subjects were healthy, nonmedicated, and free of cardiovascular abnormalities. Each subject signed an informed consent form approved by the Office of Human Research of Hiroshima University prior to participating in this study.

Experimental protocols. We designed two experiments to examine (1) the phase effects of the exercise demand (lamp on) signal on cardiac interval at the onset of exercise, and (2) the effects of training on cardiac response at the onset of exercise during maximal dynamic leg exercise.

Experiment 1. Ten subjects ( 6 females and 4 males; mean age, 21.5 years) were informed in regard to concentric contractions and were well practiced in the exercise movement using a computer-assisted isokinetic KIN/COM III dynamometer (Chattecex Corp., 101 Memorial Drive, P.O. Box 4287, Chattanooga, TN 37405) before the data were collected. The temperature in the measurement room was maintained from 24 to $26^{\circ} \mathrm{C}$.
The subjects were seated on the machine with chest, pelvis, and thighs secured by belts. The experiment was carried out on the right lower extremity. The mechanical axis of rotation was aligned with the lateral epicondyle of the femur, and the extension of the knee set at $0^{\circ}$ as the origin of knee motion. The angular velocity of voluntary movement was fixed at $180^{\circ} \%$, and the range of joint angle in the knee motion was fixed at $85^{\circ}$, from 5 to $90^{\circ}$. A $5 \mathrm{~cm}$ red lamp was placed approximately $2 \mathrm{~m}$ in front of the subject.

Each subject initiated knee extension and flexion maximally when an examiner gave the exercise demand signal (lamp). After 3 or 4 repetitions of knee flexion and extension, a verbal command was given by the examiner to stop. Each subject performed this trial 24 times, and a 3-min interval was allowed between trials to reduce fatigue. The exercise demand lamp was lit randomly to avoid anticipation.

Experiment 2. Ten subjects ( 6 females and 4 males; mean age, 21.1 years) participated in experiment 2 . The angular velocity and angle of knee motion with the KIN/COM III were the same as those in experiment 1. Eccentric contraction is defined as the contraction that occurs while the muscle length is extended $[9,10]$. When a subject extends or bends his (her) knee, KIN/COM III bends or extends the subject's knee by the servo power more than by his (her) muscular power alone. Exercise consisting of eccentric contractions is rarely used for muscle training. Each subject was told about the eccentric movement and the method of the exercise, but they did not practice the eccentric movement with the KIN/COM III beforehand. Subjects remained resting on the seat of the KIN/COM III and started knee flexion and extension as strongly as possible upon a visual command from the examiner.

One exercise set consisted of 10 continuous repetitions of voluntary maximum eccentric knee flexion and extension. A 1-min rest followed each set. Three sets of training stages per day were successively performed three times a week (Monday, Wednesday, and Friday), and stages were repeated a total of 12 times in 4 weeks. The initial increase in muscle power during a period of muscle training is caused mainly by the change in neural activity [11]. To examine the neural adaptation of the heart, a 4-week training period was decided upon.

Measurements were taken before training and after the 6th and 12th eccentric training stages. The measurements before the training and after the 6th training were regarded as the 1st and 7th trainings, respectively.

Data collection and analysis. An electrocar- 
diogram (ECG) and EMG were obtained by means of bipolar electrodes fixed on the thorax and rectus femoris, respectively (Nihon Kohden, Tokyo: bioelectric amplifier MEG-2100). The muscular torques of knee extensor and flexor movements were converted into electrical signals by the KIN/COM III. The signals of the cue lamp, ECG, EMG, and muscular torque were recorded simultaneously by using an analog data recorder (Sony, Tokyo: NFR-3000) during the rest and exercise periods (Fig. 1). EMG was not measured in experiment 2. Signals were reproduced and converted to digital data at $1,000 \mathrm{~Hz}$ by a 16 -bit analog-to-digital converter (Canopus, Kobe: ADXG-16), and data were analyzed by using wave analysis software (Canopus: Wave-Master II). The cardiac interval in which the exercise demand signal was given is defined as the "onset cardiac interval."

In experiment 1 , the onset cardiac interval was divided into three sections to assess the relation between the time from the exercise demand signal to a $\mathrm{P}$ wave and a change in the appearance of an R wave (Fig. 2). The $\mathrm{R}-\mathrm{T}, \mathrm{T}-\mathrm{P}$, and $\mathrm{P}-\mathrm{R}$ periods indicate the periods from the peak of an $R$ wave to the end of a $T$ wave, from the end of a $\mathrm{T}$ wave to the onset of a $\mathrm{P}$ wave, and from the onset of a $\mathrm{P}$ wave to the peak of an $\mathrm{R}$ wave, respectively. Twenty-four trials of each subject were divided according to the section ( $\mathrm{R}-\mathrm{T}, \mathrm{T}-\mathrm{P}$, or $\mathrm{P}-\mathrm{R}$ ) during which the visual sign to begin exercising occurred at the onset cardiac interval. After being classified into the three conditions, as described, 10 cardiac intervals before the onset cardiac interval, the onset cardiac interval, and three cardiac intervals after the onset cardiac interval were collected from each trial. An average of 10 cardiac intervals at rest was calculated, and deviations from this average and differences between a preceding and a following cardiac interval were then acquired. We also measured the time from the onset of the starting signal to the following $R$ wave at the onset cardiac interval, and the time from the onset of the starting signal to the occurrence of torque.

In experiment 2 , the exercise demand lamp was lit $80 \mathrm{~ms}$ after the voltage of the first $\mathrm{R}$ spike exceeded $500 \mathrm{mV}$. This lamp system consisted of a computer (NEC, Tokyo: 9800-DA), a digital-to-analog converter (Canopus: DAX-98), and the red lamp. After the onset cardiac interval had been identified by the position of the exercise demand signal, 10 cardiac intervals before and after the onset cardiac interval were measured, respectively. An average of the 10 intervals before the onset cardiac interval was calculated, and the deviations from this average and the differences between a preceding and a following cardiac interval were then calculated from 21 cardiac intervals.

Statistical analyses. Cardiac interval data displayed a nonparametric distribution by univariate analyses. The Wilcoxon signed rank test was used between each cardiac interval and the mean resting cardiac interval, and the Kruskal-Wallis test was used among the cardiac intervals in the three conditions ( $\mathrm{R}-\mathrm{T}, \mathrm{T}-\mathrm{P}$, and $\mathrm{P}-\mathrm{R})$ and among the cardiac intervals in the training stages (pretraining and after the 6th and 12th trainings). The level of significance was set at $p<0.05$, and data are presented as means \pm SE.

\section{RESULTS}

\section{Cardiac interval at rest and onset of exercise}

Figure 1 shows an example of changes in ECG, EMG, and muscle torque in response to the exercise demand signal. At rest, the cardiac interval estimated from the R-R interval of the ECG ranged from 736 to $914 \mathrm{~ms}$. The onset cardiac interval increased to $989 \mathrm{~ms}$ from the resting value. The resting cardiac interval, onset cardiac interval, and cardiac interval during exercise were measured from 24 trials in each of 10 subjects. The overall mean resting cardiac interval was $747 \pm 21 \mathrm{~ms}(n=2,400)$.

\section{Phasic effect of exercise demand signal in cardiac interval}

The resting cardiac interval consisted of R-T, T-P, and $\mathrm{P}-\mathrm{R}$ periods (shown on the left in Fig. 2). The $\mathrm{R}-\mathrm{T}, \mathrm{T}-\mathrm{P}$, and $\mathrm{P}-\mathrm{R}$ periods were $323 \pm 5,249 \pm 17$, and $175 \pm 5 \mathrm{~ms}$, respectively. The exercise demand signal was given at $620 \pm 22 \mathrm{~ms}$ in the $\mathrm{R}-\mathrm{T}$ period $(n=99)$, $323 \pm 17 \mathrm{~ms}$ in the T-P period $(n=73)$, and $82 \pm 12 \mathrm{~ms}$ in the $\mathrm{P}-\mathrm{R}$ period $(n=68)$ before the following $\mathrm{R}$ spike of the ECG signal (shown on the right in Fig. 2).

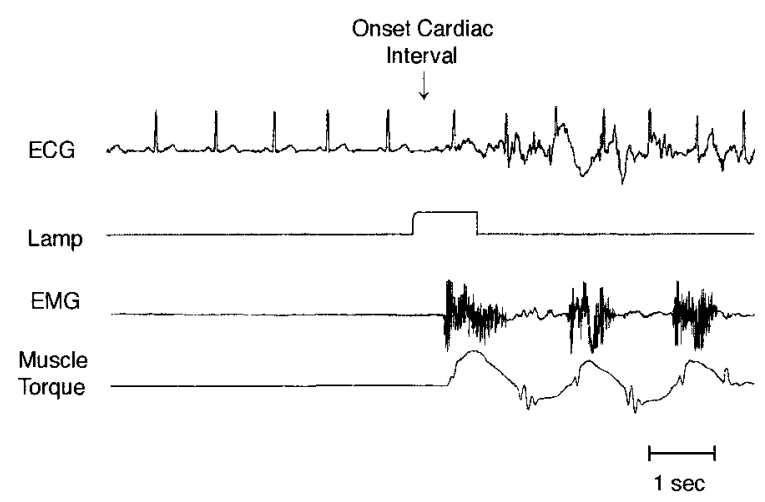

Fig. 1. Tracing of computer records from one subject showing initial ECG, EMG, and muscle torque activities to exercise started by a visual command (lamp). The onset cardiac interval is defined as the cardiac interval in which the exercise demand signal was given. 


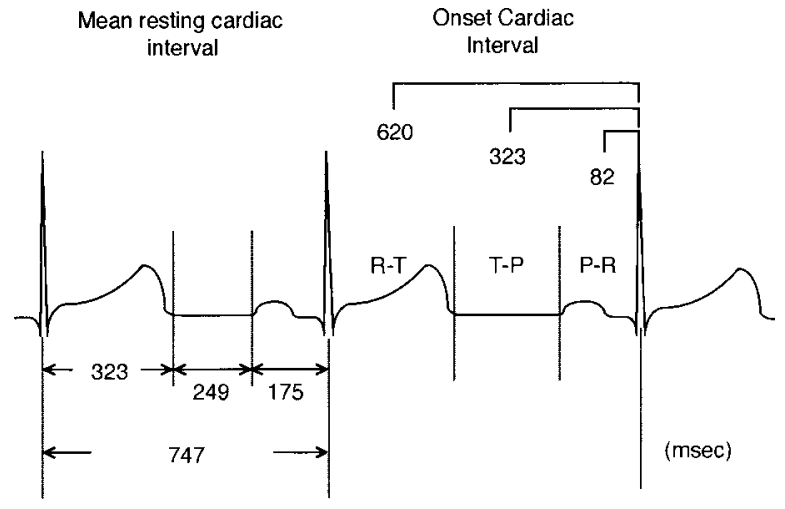

Fig. 2. Schematic diagram showing the mean resting cardiac interval and the onset cardiac interval. To examine the phasic effect of the exercise demand signal on the cardiac interval, the onset cardiac interval was divided into three sections, namely, R-T, T-P, and P-R period. The exercise demand signal was given at $620 \mathrm{~ms}$ in the R-T period, $323 \mathrm{~ms}$ in the T-P period, and $82 \mathrm{~ms}$ in the P-R period before the following $\mathrm{R}$ spike.

The EMG reaction time was defined as the delay time between the onset of exercise demand signal and the attainment of an active state in skeletal muscle. The EMG reaction time when the exercise demand signal was given in the $\mathrm{R}-\mathrm{T}, \mathrm{T}-\mathrm{P}$, and $\mathrm{P}-\mathrm{R}$ periods was $421 \pm 17,419 \pm 15$, and $424 \pm 19 \mathrm{~ms}$, respectively. Similarly, the total reaction time was defined as the time interval between the onset of exercise demand signal and the development of torque. The total reaction time after the exercise demand signal had been given in the R-T, T-P, and P-R periods was $484 \pm 21$, $484 \pm 20$, and $488 \pm 19 \mathrm{~ms}$, respectively.

Change of cardiac interval at onset and during exercise. Changes in the onset cardiac interval and the cardiac interval during exercise differed significantly depending on whether the exercise demand signal was given in the period of $\mathrm{R}-\mathrm{T}, \mathrm{T}-\mathrm{P}$, or $\mathrm{P}-\mathrm{R}$. Overall data are summarized in Fig. 3. The onset cardiac interval increased by $8 \pm 5 \mathrm{~ms}$ from the resting value only when the exercise demand signal was given in the R-T period. Changes in the onset cardiac interval were not significant statistically when the exercise demand signal was given in the $\mathrm{T}-\mathrm{P}$ and $\mathrm{P}-\mathrm{R}$ periods. The cardiac intervals during exercise decreased below the mean resting value by $55 \pm 10,79 \pm 11$, and $92 \pm$ $12 \mathrm{~ms}$ in the $1 \mathrm{st}, 2 \mathrm{nd}$, and 3rd cardiac cycles, respectively, when the exercise demand signal was given in the $\mathrm{R}-\mathrm{T}$ period. When it was given in the T-P period, the cardiac intervals during exercise decreased by $34 \pm 10,68 \pm 12$, and $88 \pm 11 \mathrm{~ms}$ in the $1 \mathrm{st}, 2 \mathrm{nd}$, and 3 rd cardiac cycles, respectively. The decreases in the 1 st and 2nd cardiac intervals during exercise were significantly larger when the exercise demand signal was

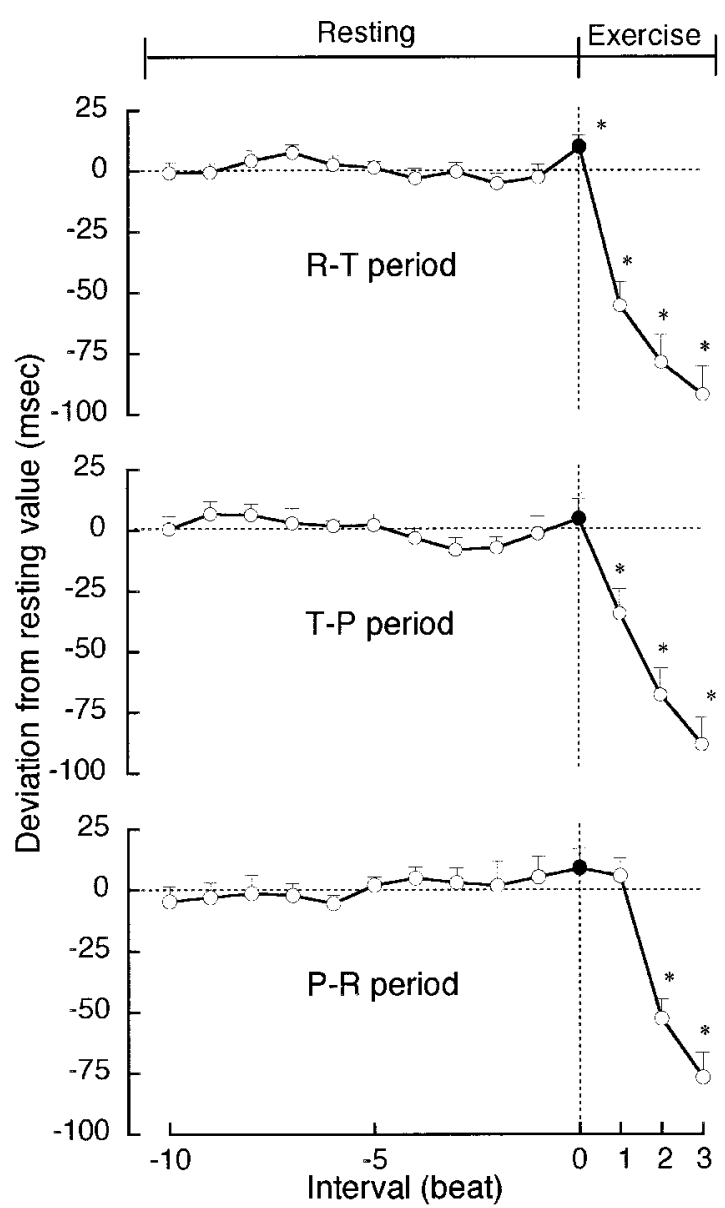

Fig. 3. Average changes in cardiac interval at rest and during the exercise when the exercise demand signal was given in the R-T (upper), T-P (middle), and P-R period (lower). The filled circle indicates the onset cardiac interval. The values are means \pm SE. * Significantly different from the mean resting value, $p<0.05$. 'Significantly different from the mean resting value, $p<0.01$.

begun in the R-T period than when it was given in the $\mathrm{T}-\mathrm{P}$ period. When the exercise demand signal was given in the $\mathrm{P}-\mathrm{R}$ period, the cardiac interval during exercise did not decrease in the 1 st cardiac cycle. It decreased by $53 \pm 8 \mathrm{~ms}$ in the 2 nd cardiac cycle and by $77 \pm 10 \mathrm{~ms}$ in the $3 \mathrm{rd}$ cardiac cycle. When the exercise demand signal was given in the P-R period, the decreases in the 2 nd and 3rd cardiac intervals during exercise were significantly smaller compared with those in the $\mathrm{R}-\mathrm{T}$ and $\mathrm{T}-\mathrm{P}$ periods.

Beat-to-beat changes in cardiac interval. We analyzed the changes in the cardiac interval in response to the exercise demand signal on a beat-to-beat basis. Figure 4 shows the summarized data obtained when the exercise demand signal was given in the $\mathrm{R}-\mathrm{T}$, $\mathrm{T}-\mathrm{P}$, and $\mathrm{P}-\mathrm{R}$ periods. When the exercise demand signal was given in the $\mathrm{R}-\mathrm{T}, \mathrm{T}-\mathrm{P}$, and $\mathrm{P}-\mathrm{R}$ periods, the magnitude of decreases in the cardiac interval in the 1st and 2nd cardiac cycles during exercise dif- 


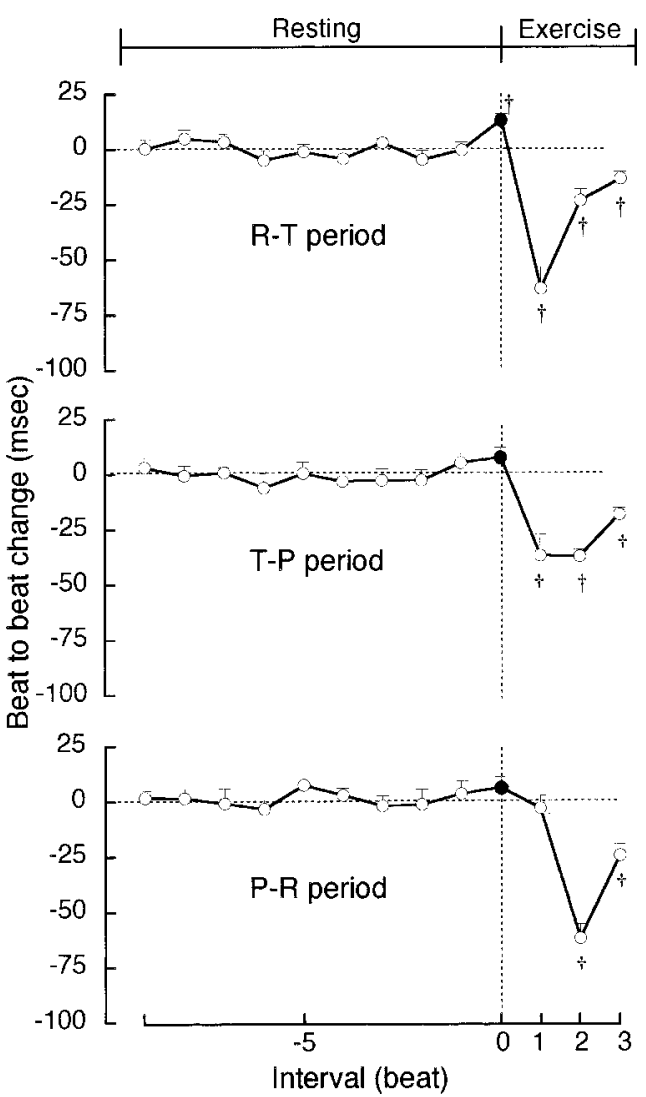

Fig. 4. Beat-to-beat changes in cardiac interval at rest and during exercise when the exercise demand signal was given in the R-T (upper), T-P (middle), and P-R period (lower). The filled circle indicates the onset cardiac interval. Values are means \pm SE. ${ }^{\dagger} p<0.01$.

fered significantly among the three measurement periods. Decreases in the 1 st cardiac interval from the onset cardiac interval were $63 \pm 10 \mathrm{~ms}$ for the $\mathrm{R}-\mathrm{T}$ period, $37 \pm 10 \mathrm{~ms}$ for the $\mathrm{T}-\mathrm{P}$ period, and $3 \pm 6 \mathrm{~ms}$ for the $\mathrm{P}-\mathrm{R}$ period. The decrease in the 1 st cardiac interval in the $\mathrm{R}-\mathrm{T}$ period was larger than that in the $\mathrm{T}-\mathrm{P}$ period. No significant decrease in the 1st cardiac interval was observed in the $\mathrm{P}-\mathrm{R}$ period. The decreases in the 2nd cardiac interval during the exercise from the 1 st cardiac interval were $23 \pm 5,37 \pm 3$, and $62 \pm 6$ $\mathrm{ms}$ for demand signals in the R-T, T-P, and P-R periods, respectively. Differences in the decrease in the 2nd cardiac interval were statistically significant among the three periods.

\section{Training effects on cardiac interval}

The resting cardiac interval was $811 \pm 14(n=300)$, $779 \pm 13(n=300)$, and $744 \pm 13 \mathrm{~ms}(n=300)$ in pretraining, and after the 6th and the 12th training stages, respectively. The differences in resting cardiac intervals among the three stages were statistically significant. The mean resting cardiac interval varied among different subjects, and therefore the overall mean

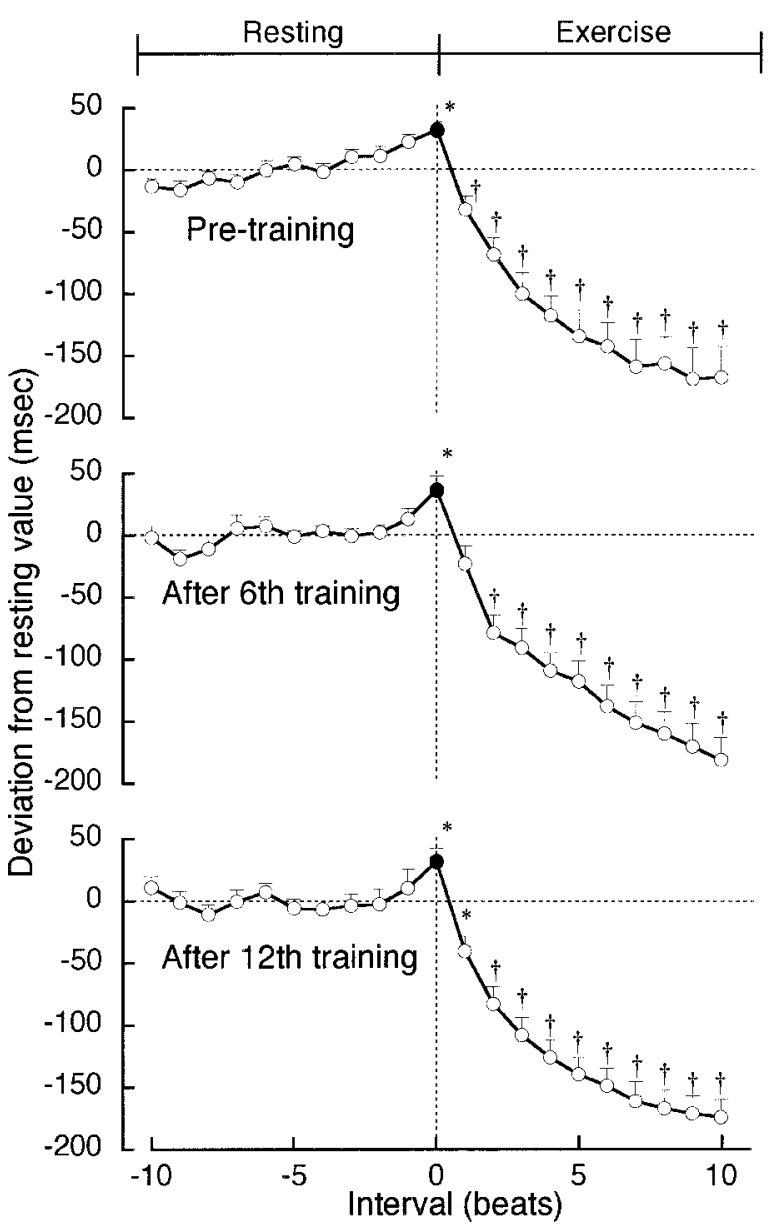

Fig. 5. Average changes in the cardiac interval at rest and during exercise in pretraining (upper), after the 6th training stage (middle), and after the 12th training stage (lower). The filled circle indicates the onset cardiac interval. The values are means \pm SE. ${ }^{*} p<0.05 ;{ }^{\dagger} p<0.01$.

value was set as zero; the deviation of the cardiac interval from the overall mean value was obtained at rest and during exercise in the pretraining and after the 6th and 12th training stages (Fig. 5).

The onset cardiac intervals increased from the mean resting value by $32 \pm 6,36 \pm 11$, and $32 \pm 10 \mathrm{~ms}$ in pretraining, after the 6th training stage, and after the 12th training stage, respectively. The increases in the onset cardiac interval were almost the same in the three stages. During exercise, the cardiac interval decreased rapidly and below the mean resting value. The magnitude of decrease in the cardiac interval measured from the 10 cardiac cycles during exercise was $-124 \pm 10,-121 \pm 9$, and $-131 \pm 8 \mathrm{~ms}$ in pretraining, after the 6th training stage, and after the 12th training stage, respectively. The differences in response magnitudes among the three stages were not significant. The magnitude of decrease in the 10th cardiac cycle was $167 \pm 25,181 \pm 18$, and $173 \pm 14 \mathrm{~ms}$ in pretraining, after the 6th training stage, and after the 12th training 


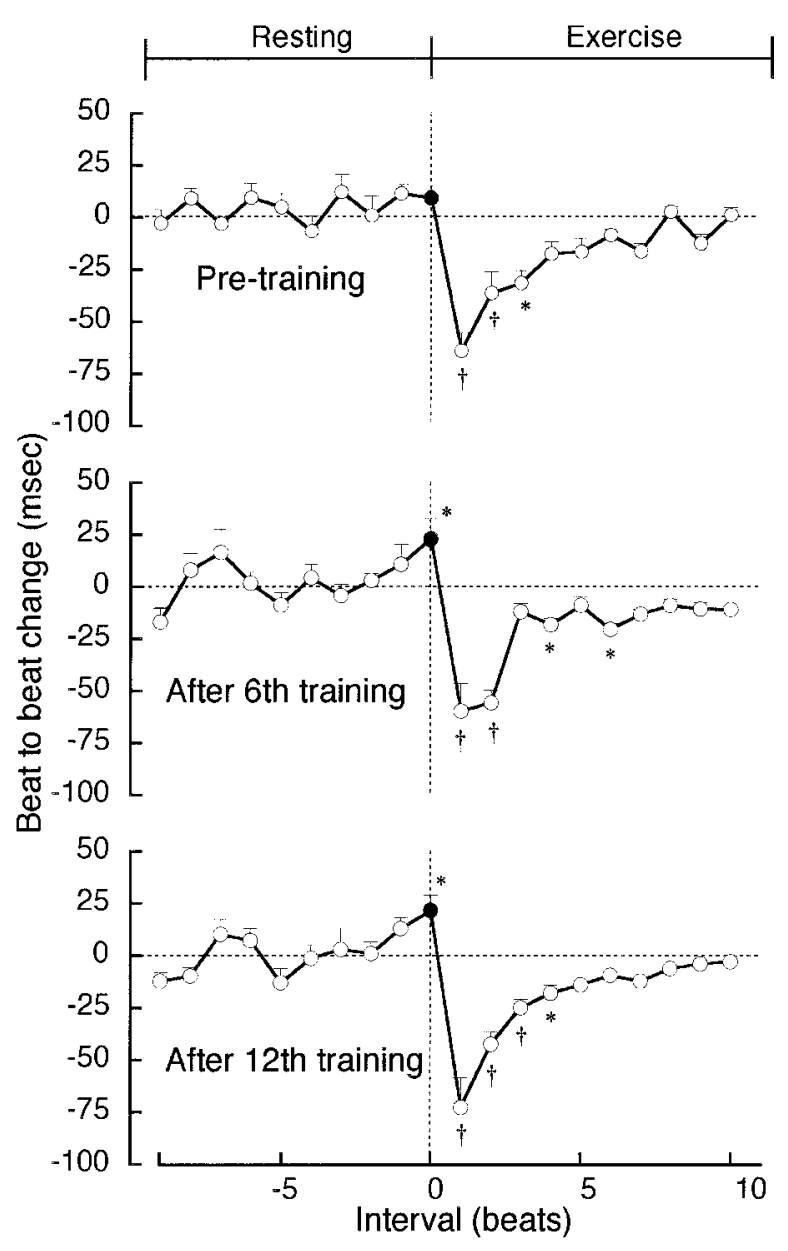

Fig. 6. Beat-to-beat changes in cardiac interval at rest and during exercise in pretraining (upper), after the 6th training stage (middle), and after the 12th training stage (lower). The filled circle indicates the onset cardiac interval. The values are means \pm SE. ${ }^{*} p<0.05 ;{ }^{\dagger} p<0.01$.

stage, respectively.

To analyze the time course of response, the beat-tobeat change in the cardiac interval was obtained in the three stages (Fig. 6). The difference in changes between the onset cardiac interval and the 1st cardiac interval just after the onset cardiac interval was $-63 \pm 7$, $-62 \pm 12$, and $-73 \pm 10 \mathrm{~ms}$ in pretraining, after the 6 th training stage, and after the 12th training stage, respectively. The difference in changes in cardiac interval between pretraining and after the 12th training stage was significant.

\section{DISCUSSION}

Phase effect on cardiac interval at onset and during exercise. In this study, we attempted to evaluate the effects of the exercise demand signal and of exercise of the lower extremity on the cardiac interval in healthy human subjects. We found that the cardiac interval was increased immediately by the ex-

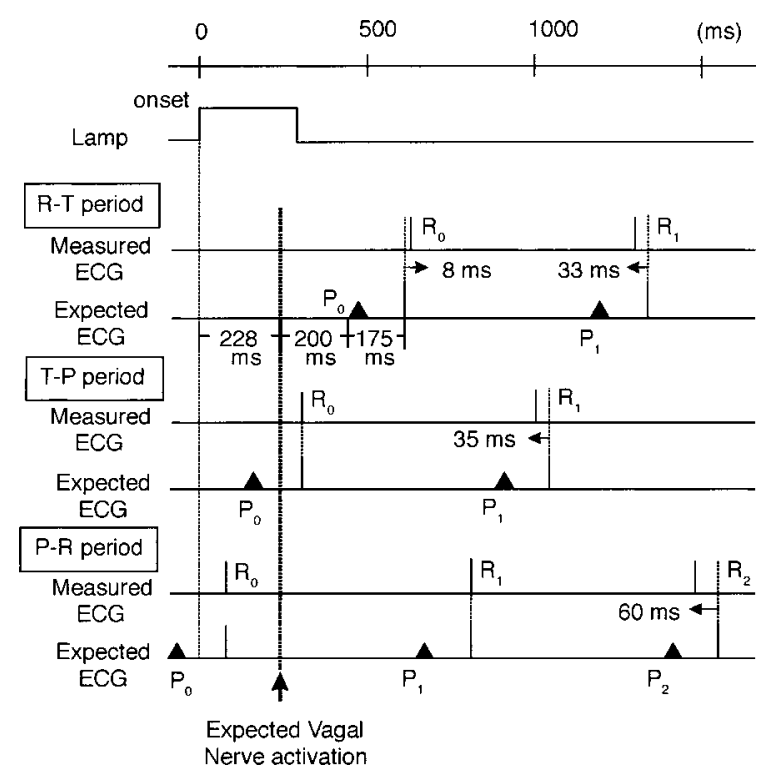

Fig. 7. Schema of time relationship during beginning of the lamp signal, the measured ECG, and expected ECG when the exercise demand signal is in the $R-T$, $\mathbf{T}-\mathbf{P}$, and $\mathbf{P}-\mathbf{R}$ intervals. The arrow and the heavy vertical dotted line show the expected vagal nerve activation, which reflects the time limit for vagal nerve activity to lengthen the $\mathrm{R}_{0}$ spike in the $\mathrm{R}-\mathrm{T}$ period.

ercise demand signal itself. This finding suggests that the vagal nerve activity in the pacemaker region should increase in response to the exercise demand signal and thus cause an increase in the cardiac interval. When the exercise demand signal was given during the $\mathrm{T}-\mathrm{P}$ or $\mathrm{P}-\mathrm{R}$ period, however, the cardiac interval did not increase.

To provide a better understanding of the mechanisms producing the difference in the initial cardiac responses, we have summarized the data obtained in this experiment and their time relationships to the onset of exercise demand signal, and we schematically show the measured and the expected ECGs in Fig. 7. The timing of R spikes in the expected ECG is superimposed on a thin dotted line in the measured ECG.

When the exercise demand signal was given in the $\mathrm{R}-\mathrm{T}$ period, the measured $\mathrm{R}_{0}$ spike appeared $8 \mathrm{~ms}$ later in comparison with the expected $\mathrm{R}_{0}$. This finding indicates that the ventricular depolarization was delayed because of vagal nerve activation when the onset of exercise demand signal was given at $428 \mathrm{~ms}$ before the expected $\mathrm{P}_{0}$. On the other hand, when the onset was given at $144 \mathrm{~ms}$ before $\mathrm{P}_{0}(\mathrm{~T}-\mathrm{P}$ period) or at $647 \mathrm{~ms}$ before $\mathrm{P}_{1}$ (P-R period), no delay of $\mathrm{R}_{0}$ or of $\mathrm{R}_{1}$ was induced. These findings suggest that the timing of vagal nerve activation before ventricular depolarization is important in causing the increase in cardiac interval in response to the exercise demand sig- 
nal. Levy et al. [12] reported that when the vagal nerve is stimulated electrically in anesthetized dogs, the latent period between vagal stimuli and the next atrial depolarization can be longer than $200 \mathrm{~ms}$. If we employed this value as a latent period for humans in this study, the vagal nerve activation had to occur within $228 \mathrm{~ms}$ after the onset of exercise demand signal (indicated by a heavy dotted line in Fig. 7). In T-P period stimulation, the time interval of $144 \mathrm{~ms}$ from the onset of exercise demand signal to $\mathrm{P}_{0}$ was not sufficient to cause a delay of $\mathrm{P}_{0}$ or $\mathrm{R}_{0}$. It is generally believed that the decrease in cardiac interval during exercise is induced by a decease in vagal nerve activity and/or by an increase in sympathetic nerve activity. The cardiac sympathetic nerve stimulation shortens the cardiac interval after a latent period of about $1-3 \mathrm{~s}$, although the vagal nervous stimulation or cessation rapidly changes the cardiac interval [13]. In the $\mathrm{R}-\mathrm{T}$ period stimulation, a $41 \mathrm{~ms}$ decrease in the $\mathrm{R}_{0}-\mathrm{R}_{1}$ cardiac interval was produced by an $8 \mathrm{~ms}$ delay of $\mathrm{R}_{0}$ and a $33 \mathrm{~ms}$ earliness of the $\mathrm{R}_{1}$ (Fig. 7). This result suggested that the decrease in cardiac interval $\left(\mathrm{R}_{0}-\mathrm{R}_{1}\right)$ was induced by a delay of ventricular depolarization because of vagal activation and by an earliness of ventricular depolarization because of vagal withdrawal and/or sympathetic activation.

In $\mathrm{T}-\mathrm{P}$ stimulation, the $\mathrm{R}_{0}-\mathrm{R}_{1}$ interval decreased. This result suggests that the decrease in cardiac interval occurred because of the effect of vagal withdrawal after the effect of brief vagal activation. In P-R stimulation, no change in the $R_{0}-R_{1}$ interval suggests that the increased effect of brief vagal activation was cancelled by the decreased effect of vagal inhibition. When a contraction is made with leg extension, the cardiovascular response is dominated by a concomitantly performed Valsalva-like maneuver [14]. An inadvertent Valsalva-like maneuver will have significant influence on the blood pressure, but not on the heart rate response to static exercise. Although the subjects in this study performed a dynamic muscle contraction, we assume that a part of the response to the Valsalvalike maneuver may have contributed to controlling the rapid increase in the cardiac interval at the onset of exercise.

Obrist [15] reviewed the cardiac-somatic relationship and noted that paradoxical heart rate changes have been observed in human subjects under conditions such as anticipation of averse stimulus [16], sensory intact tasks [17], and those relating to response execution using a signaled reaction time task [18]. The human heart rate decelerates in anticipation of any motivationally or emotionally significant event $[19,20]$. The biphasic anticipatory response consist- ing of an initial brief acceleration of heart rate followed by a more pronounced and sustained deceleration is the product of vagal influences, namely, first a decrease for $6 \mathrm{~s}$, then an increase for $9 \mathrm{~s}$ in vagal tone [21]. These reports suggest that the brief increase in cardiac interval at the onset of exercise was not elicited by a mental reaction such as anxiety or uneasiness.

Comparison of response times between heart and skeletal muscle. We compared the response times between the autonomic and somatic nervous systems under the condition of starting with a visual command. The onset time of EMG as the somatic controlling signal appeared two times later compared with the onset time of the expected vagal nerve activation, and both nerve activations seemed to be independent of each other. This result suggests that in response to the onset of exercise demand signal, the neural control signal of the pacemaker and atrium was generated before the neural control signal of skeletal muscle, and that the cardiac response to the exercise demand signal appeared more quickly than the skeletal muscle response did under the maximum muscular contraction.

Training effects of the cardiac interval. Generally, exercise training decreases a resting heart rate. But in this study the resting heart rates increased with the training. We assumed that the subjects' resting heart rates increased with repeated training of eccentric contraction to effect a transition to higher heart rate levels as quickly and smoothly as possible to respond to maximal dynamic leg exercise.

It is generally agreed that the resting heart rate is determined by a balance between sympathetic and vagal nerve activities. In human subjects, when the effects of both nerve activities are blocked, the resting heart rate increases; therefore, the vagal effect on the heart rate is dominant in comparison with the sympathetic effect. The heart rate during simultaneous stimulation of both nerves is slightly higher than that during vagal stimulation alone $[13,22]$. We speculated that in the present experiment the training experience caused increases in the resting heart rates by means of increases in the resting sympathetic nerve activity, which facilitated preparation for the maximal dynamic leg exercise. Exercise training involving eccentric contraction produces a greater increase in muscular strength than do other exercise forms involving isometric or concentric contraction [23-25]. Increases in muscular strength after 3-4 weeks of muscle training in particular are caused mainly not by muscular hypertrophy, but by the increase of neural activity [11].

Ninomiya and Wilson [26] reported that at the tran- 
sition phase from the onset of exercise to the steady state during exercise, the time course of the heart rate response curve was significantly influenced by the experience of exercise trials, but the magnitude of the increase of heart rate at the steady state during exercise remained constant and therefore independent of the experience of the exercise trials. In agreement with these results obtained in dogs, we obtained similar results in human subjects. The time course of responses in the cardiac interval was modified by the exercise training. In Fig. 5, the time course of cardiac interval response showed a smooth pattern after the 12th training stage in comparison with the pretraining, and the variation of curves diminished, reflecting small standard error. On the other hand, the magnitude of the decreased cardiac interval at 10 cardiac cycles during exercise remained almost constant, independent of the number of training trials. The neural learning effect on the magnitude of cardiac response was not recognized under the condition of repeated maximum eccentric contraction of the lower extremities.

In our experiments it was shown that (1) the brief increase in the cardiac interval at the onset of exercise occurred only when the exercise demand signal was given in the R-T period, (2) the timing between neural activity to the heart and that to the skeletal muscles at the onset of exercise differs, (3) the brief increase in the cardiac interval at the onset of exercise did not change with the repeated maximum eccentric training, and (4) the time course of the heart rate response curve was influenced, but the magnitude of the increase in heart rate during the exercise was not influenced by the training.

\section{REFERENCES}

1. Freyschuss $U$ : Elicitation of heart rate and blood pressure increase on muscle contraction. J Appl Physiol 28: $758-761,1970$

2. Christensen NJ and Brandsborg O: The relationship between plasma catecholamine concentration and pulse rate during exercise and standing. Eur $\mathrm{J}$ Clin Invest 3: 299-306, 1973

3. Fagraeus $L$ and Linnarsson $D$ : Autonomic origin of heart rate fluctuations at the onset of muscular exercise. J Appl Physiol 40: 679-682, 1976

4. Robinson BF, Epstein SE, Beiser GD, and Braunwald E: Control of heart rate by the autonomic nervous system. Circ Res 19: 400-411, 1966

5. Yamamoto Y, Hughson RL, and Peterson JC: Autonomic control of heart rate during exercise studied by heart rate variability spectral analysis. J Appl Physiol 71: 1136-1142, 1991

6. Sun JC, Eiken O, and Mekjavic IB: Autonomic nervous control of heart rate during blood-flow restricted exercise in man. Eur J Appl Physiol 66: 202-206, 1993

7. Hollander AP and Bouman LN: Cardiac acceleration in man elicited by a muscle-heart reflex. J Appl Physiol 38: 272-278, 1975

8. Takayanagi K, Yoshimura O, and Ninomiya I: Effects of repetitive physical training on neurogenic regulation of heart rate in healthy subjects. J Physiol Soc Jpn 59: 139-154, 1997

9. Asmussen E: Positive and negative muscular work. Acta Physiol Scand 28: 364-382,1953

10. Aura $\mathrm{O}$ and Komi PV: Mechanical efficiency of pure positive and pure negative work with special reference to work intensity. Int J Sports Med 7: 44-49, 1986

11. Weiss LW and Clark FC: Ultrasonic measurement of upper-arm skeletal muscle thickness. J Sports Med 27: 128-133, 1987

12. Levy MN, Martin PJ, lano T, and Zieske T: Effects of single stimuli on heart rate and atrioventricular condition. Am J Physiol 218: 1256-1262, 1970

13. Warner HR and Cox A: A mathematical model of heart rate control by sympathetic and vagus efferent information. J Appl Physiol 17: 349-355, 1962

14. Lassen A, Mitchell JH, Reeves DRJ, Rogers HB, and Secher NH: Cardiovascular responses to brief static contractions in man with topical nervous blockade. J Physiol (Lond) 409: 333-341, 1989

15. Obrist PA: Cardiovascular Psychophysiology, Plenum Press, New York, pp 47-81, 1981

16. Notterman JM, Schoenfeld WN, and Bersh PJ: Conditioned heart rate responses in human beings during experimental anxiety. J Comp Physiol Psychol 45: 1-8, 1952

17. Obrist PA: Cardiovascular differentiation of sensory stimuli. Psychosom Med 25: 450-458, 1963

18. Lacey Jl: Somatic response patterning and stress: some revisions of activation theory. In: Psychological Stress: Issues in Research, ed. Appley $\mathrm{MH}$ and Trumbull R, Appleton-Century-Crofts, New York, pp 14-38, 1967

19. Geer JH: Measurement of the conditioned cardiac response. J Comp Physiol Psychol 57: 426-433, 1964

20. Wilson RN: Cardiac response determinants of conditioning. J Comp Physiol Psychol 68: 1-23, 1969

21. Obrist PA, Wood DM, and Perez-Reyes M: Heart rate during conditioning in humans: effects of UCS intensity, vagal blockade and adrenergic block of vasomotor activity. J Exp Psychol 70: 32-42, 1965

22. Levy MN and Zieske $\mathrm{H}$ : Autonomic control of cardiac pacemaker activity and atrioventricular transmission. J Appl Physiol 27: 465-470, 1969

23. Komi PV and Buskirk ER: Effect of eccentric and concentric muscle conditioning on tension and electrical activity of human muscle. Elgonomics 15: 417-434, 1972

24. Duncan PW, Chandler JM, Cavanaugh DK, Johnson $K R$, and Buehler AG: Mode and speed specificity of eccentric and concentric exercise training. J Orthop Sports Phys Ther 9: 456-462, 1989

25. Takayanagi $\mathrm{K}$, Yoshimura $\mathrm{O}$, Ihara $\mathrm{H}$, and Nakayama $A$ : Velocity and mode specificity of concentric and eccentric training in knee flexors and extensors. J Phys Ther Sci 7: 57-63, 1995

26. Ninomiya I and Wilson MF: Cardiac adaptation at the transition phases of exercise in unanesthetized dogs. J Appl Physiol 21: 953-958,1966 